\title{
The influence of transketolase on lipid biosynthesis in the yeast Yarrowia lipolytica
}

\author{
Adam Dobrowolski* (i) and Aleksandra M. Mirończuk(1)
}

\begin{abstract}
Background: During the pentose phosphate pathway (PPP), two important components, NADPH and pentoses, are provided to the cell. Previously it was shown that this metabolic pathway is a source of reducing agent for lipid synthesis from glucose in the yeast Yarrowia lipolytica. Y. lipolytica is an attractive microbial host since it is able to convert untypical feedstocks, such as glycerol, into oils, which subsequently can be transesterified to biodiesel. However, the lipogenesis process is a complex phenomenon, and it still remains unknown which genes from the PPP are involved in lipid synthesis.
\end{abstract}

Results: To address this problem we overexpressed five genes from this metabolic pathway: transaldolase (TAL1, YAL1OF15587g), transketolase (TKL1, YALIOE06479g), ribulose-phosphate 3-epimerase (RPE1, YALIOC11880g) and two dehydrogenases, NADP+ ${ }^{+}$-dependent glucose-6-phosphate dehydrogenase (ZWF1, YALIOE22649g) and NADP+ ${ }^{+}$-dependent 6-phosphogluconate dehydrogenase (GND1, YAL1OB15598g), simultaneously with diacylglycerol acyltransferase (DGA1, YALIOE32769g) and verified each resulting strain's ability to synthesize fatty acid growing on both glycerol and glucose as a carbon source. Our results showed that co-expression of DGA1 and TKL1 results in higher SCO synthesis, increasing lipid content by $40 \%$ over the control strain (DGA1 overexpression).

Conclusions: Simultaneous overexpression of DGA1 and TKL1 genes results in a higher lipid titer independently from the fermentation conditions, such as carbon source, $\mathrm{pH}$ and $\mathrm{YE}$ supplementation.

Keywords: Pentose phosphate pathway (PPP), Lipids, Yarrowia lipolytica, Transketolase

\section{Background}

Nowadays, most global industry is dependent on fossil fuels. Depletion of this source forces scientists to develop alternative substrates for biodiesel feedstock. A suitable replacement for this is biodiesel derived from plants such as sunflowers or canola. Unfortunately, its production requires a huge amount of freshwater and areas of farmlands, which can be applied for the food industry. Thus a decreasing amount of freshwater available for agriculture industry causes that biodiesel derived from vegetable oils becomes ethically doubtful.

*Correspondence: adam.dobrowolski@upwr.edu.pl

Department of Biotechnology and Food Microbiology, Wroclaw University of Environmental and Life Sciences, Chełmońskiego 37,

51-630 Wrocław, Poland
For this reason a single cell oil (SCO) derived from microbial biomass is a promising replacement for biodiesel in the coming decades. One of the suitable producers of biodiesel precursors is Yarrowia lipolytica, an unconventional yeast that is able to produce lipids over $30 \%$ of its dry biomass $[1,2]$ from untypical carbon sources such as alkanes, glycerol or agricultural wastes [3-5]. This well-studied yeast possesses a fully sequenced genome and well-developed genetic tools, including CRISPR/Cas9 [6-8]. Its metabolic engineering or adaptive laboratory evolution (ALE) allowed for the modification of the fatty acid profile, content and employment of a wide range of substrates [9-12]. Moreover, this yeast is able to grow at low $\mathrm{pH}$ and use seawater [13], which is an advantage during the fermentation processes on the industrial scale. Lipogenesis 
is a natural process that occurs in $Y$. lipolytica during nitrogen starvation. Under these conditions, the cells start to accumulate fatty acids in the lipid bodies. The metabolic pathway of lipid synthesis in Y. lipolytica has been well studied. A few key factors have been identified in this process, namely malic enzyme (ME), ATP citrate lyase (ACL), acetyl-CoA carboxylase (ACC), glycerol-3-P-O-acyltransferase (SCT1), 1-acylglycerol-P-acyltransferase (SLC1), diacylglycerol (DAG) acyltransferase (DGA1 or DGA2) and phospholipid DAG acyltransferase (LRO1) [2]. In particular, it was shown that simple overexpression of the DGA1 (YALIOE32769g) gene results in elevated lipid synthesis by Y. lipolytica [14]. Moreover, this process required an increased level of NADPH that is provided by $\mathrm{NADP}^{+}$-dependent isocitrate dehydrogenase, the oxidative pentose pathway (oxPPP) or malic enzyme [15].

The aim of this study was to improve lipid synthesis in Y. lipolytica by co-expression of the DGA1 (YALIOE32769g) gene and the genes involved in PPP, transaldolase (TAL1, YALIOF15587g), transketolase (TKL1, YALIOE06479g), ribulose-phosphate 3-epimerase (RPE1, YALI0C11880g) and two dehydrogenases, ZWF1 (YALIOE22649g) and GND1 (YALIOB15598g). Next, we compared the productivity of the process using two different carbon sources: glycerol and glucose. Finally, the influence of the $\mathrm{pH}$ on the engineered strain was tested. The highest lipid synthesis was observed during simultaneous overexpression of DGA1 and transketolase (TKL1) genes.

\section{Methods}

\section{Microorganisms}

Strains used in this study are listed in Table 1. These strains belong to the Department of Biotechnology and Food Microbiology at Wroclaw University of Environmental and Life Sciences, Poland.

\section{Media and culture conditions}

Medium LB (BTL, Poland) was used for cultivation of Escherichia coli strains. The inoculum of yeast strains was prepared in Rich Yeast Extract Peptone Glucose (YPD) and it contained: $10 \mathrm{~g} / \mathrm{L}$ yeast extract (Merck, Germany), $10 \mathrm{~g} / \mathrm{L}$ peptone (Biocorp, Poland) and $20 \mathrm{~g} / \mathrm{L}$ glucose (Merck, Germany). The medium for the lipid production consisted of: Medium A: YNB (without amino acids and ammonium sulfate, Sigma, Germany), $50 \mathrm{~g} / \mathrm{L}$ pure glycerol (POCH, Poland) supplemented with $\left(\mathrm{NH}_{4}\right)_{2} \mathrm{SO}_{4}$, ratio $\mathrm{C} / \mathrm{N}$ 60, pH 6.0; Medium B: YNB (w/o aa, w/o ammonium sulfate), $50 \mathrm{~g} / \mathrm{L}$ pure glycerol, supplemented with $\left(\mathrm{NH}_{4}\right)_{2} \mathrm{SO}_{4}, 0.5 \mathrm{~g} / \mathrm{L} \mathrm{YE}$, ratio $\mathrm{C} / \mathrm{N}$ 60, pH 3.0, maintained by $50 \mathrm{mM}$ citrate buffer; Medium C: YNB (w/o aa, w/o ammonium sulfate), $50 \mathrm{~g} / \mathrm{L}$ glucose (Merck, Germany), supplemented with $\left(\mathrm{NH}_{4}\right)_{2} \mathrm{SO}_{4}, 0.5 \mathrm{~g} / \mathrm{L} \mathrm{YE}$, ratio $\mathrm{C} / \mathrm{N} 60$, $\mathrm{pH}$ 3.0, maintained by $50 \mathrm{mM}$ citrate buffer.

\section{Analytical methods}

$10 \mathrm{~mL}$ of samples were spun down $\left(10 \mathrm{~min} ; 4{ }^{\circ} \mathrm{C}\right.$; $5500 \times g$ ), then filtered on $0.45-\mu \mathrm{m}$ pore membranes and washed twice with distilled water. After drying at $105^{\circ} \mathrm{C}$, the biomass was determined gravimetrically. The fatty acids (FAs) from lyophilized biomass were derivatized

Table 1 Strains used in this study

\begin{tabular}{|c|c|c|}
\hline Strain & Genotype or plasmid & Source \\
\hline \multicolumn{3}{|l|}{ E. coli } \\
\hline $\mathrm{DH} 5 \mathrm{a}$ & $\begin{array}{l}\mathrm{F}^{-} \text {endA1 glnV44 thi-1 recA1 relA1 gyrA96 deoR nupG } \$ 80 \text { dlacZ } \triangle M 15 \triangle(\text { lacZYA-argF)U169, } \\
\text { hsdR17(rK- mK }+ \text { ), } \lambda-\end{array}$ & [16] \\
\hline $\mathrm{DH} 5 \mathrm{a}$ & pAD-RPE1, YALIOC11880g & This study \\
\hline $\mathrm{DH} 5 \mathrm{a}$ & pAD-TKL1, YALIOE06479g & [16] \\
\hline $\mathrm{DH} 5 \mathrm{a}$ & PAD-TAL1, YALIOF15587g & [16] \\
\hline $\mathrm{DH} 5 \mathrm{a}$ & pAD-GDN1, YALIOB15598g & [16] \\
\hline $\mathrm{DH} 5 \mathrm{a}$ & pAD-ZWF1, YALIOE22649g & [16] \\
\hline $\mathrm{DH} 5 \mathrm{a}$ & pAD-DGA1, YALIOE32769g & [13] \\
\hline \multicolumn{3}{|l|}{ Y. lipolytica } \\
\hline AJD & MATA, AJD: ura3-302 & [18] \\
\hline AJD pAD-DGA1 & MATA, AJD ura3-302, overexpression YALIOE32769g & [13] \\
\hline AJD D/TKL1 & MATA, AJD: ura3-302, overexpression YALIOE32769g,YALIOE06479g & This study \\
\hline AJD D/TAL1 & MATA, AJD: ura3-302, overexpression YALIOE32769g, YALIOF15587g & This study \\
\hline AJD D/RPE1 & MATA, AJD: ura3-302, overexpression YALIOE32769g, YALIOC11880g & This study \\
\hline AJD D/GDN1 & MATA, AJD: ura3-302, overexpression YALIOE32769g, YALIOB15598g & This study \\
\hline AJD D/ZWF1 & MATA, AJD: ura3-302, overexpression YALIOE32769g, YALIOE22649g & This study \\
\hline
\end{tabular}


to fatty acid methyl esters (FAMEs) using the method described before [13]. FAMEs were analyzed by gas chromatography on GC-2010 Plus apparatus (Shimadzu, Japan) with a flame ionization detector (FID) and autoinjector (AOC-20i). The separation of FAMEs was achieved using a 70\% cyanopropyl polysilphenylene-siloxane column (TR-FAME, $30 \mathrm{~m} \times 0.32 \mathrm{~mm} \times 0.25 \mu \mathrm{m}$ ). The initial oven temperature was $130{ }^{\circ} \mathrm{C}$ held for $1 \mathrm{~min}$, which was then increased to $200{ }^{\circ} \mathrm{C}$ at the rate of $5{ }^{\circ} \mathrm{C} \times \mathrm{min}^{-1}$, then increased to $250{ }^{\circ} \mathrm{C}$ at a rate of $10{ }^{\circ} \mathrm{C} \times \mathrm{min}^{-1}$ and held for $1 \mathrm{~min}$. Temperature for the injector and detector were $270{ }^{\circ} \mathrm{C}$ and $280{ }^{\circ} \mathrm{C}$, respectively. Helium was used as the carrier gas with constant flow $1.52 \mathrm{~mL} \times \mathrm{min}^{-1}$. Volume of injection was $1 \mu \mathrm{L}$ with a split rate of 1:5. The identification of FAME was evaluated using Supelco 37 Component Fame Mix as a reference standard and for quantification analysis heptanoic acid was used as an internal standard. The total lipid content in dry cell weight was calculated as the sum of all fatty acids.

\section{Spark microplate reader (TECAN)}

The yeast strain was grown in 96-well plates in $200 \mu \mathrm{L}$ of YNB medium supplemented with 5\% glycerol. First, the strains were grown for $24 \mathrm{~h}$ in the same medium, then the cultures were spun down, washed with sterile water and inoculated to an initial $\mathrm{OD}_{600}$ of 0.1 in each well. Three biological replications were used in this experiments. The strains were grown at $28^{\circ} \mathrm{C}$ under constant agitation with a SPARK microplate reader (TECAN). Growth was monitored measuring optical density at $\lambda_{600}$ every $30 \mathrm{~min}$ for $24 \mathrm{~h}$.

\section{Shake-flask experiments}

The inoculum was grown in YPD medium. Three production media for the shake-flask experiment were used in the study. Production Medium A consisted of: YNB and pure $50 \mathrm{~g} / \mathrm{L}$ glycerol, $\left(\mathrm{NH}_{4}\right)_{2} \mathrm{SO}_{4}$ to $\mathrm{C} / \mathrm{N}$ ratio 60 , $\mathrm{pH}$ 6.0. Production Medium B consisted of: YNB and pure $50 \mathrm{~g} / \mathrm{L}$ glycerol, $(\mathrm{NH} 4)_{2} \mathrm{SO}_{4}$ to $\mathrm{C} / \mathrm{N}$ ratio $60, \mathrm{pH}$ 3.0, maintained by addition of citrate buffer. Production Medium C consisted of: YNB and pure $50 \mathrm{~g} / \mathrm{L}$ glucose, $\left(\mathrm{NH}_{4}\right)_{2} \mathrm{SO}_{4}$ to $\mathrm{C} / \mathrm{N}$ ratio $60, \mathrm{pH} 3.0$, maintained by citrate buffer $(50 \mathrm{mM})$. During shake-flask experiments the cultures were grown in $0.3 \mathrm{~L}$ flasks containing $0.03 \mathrm{~L}$ of medium on a rotary shaker (CERTOMAT IS, Sartorius Stedim Biotech) at $28^{\circ} \mathrm{C}$ at $200 \mathrm{rpm}$ for $120 \mathrm{~h}$.

\section{Construction of the RPE1 overexpressing strain}

First, the RPE1 gene (YALIOC11880g) was amplified using primers RPE1-SgSI-F (5'-ATCGGCGCGCCATGGTCC AGCCAATCATC-3) and RPE1-NheI-R (5'-CTAGCT AGC TGCCGCCTGATtAgGCAG-3). The obtained 780 bp PCR product was digested with SgSI and NheI and cloned into the corresponding site in the pAD vector [16]. The sequenced plasmid was digested with MssI enzyme and subsequently transformed into the strain $Y$. lipolytica AJD pAD-DGA1(ura-). The integration in the genome was checked by three independent PCRs.

\section{RNA isolation and transcript quantification}

The cultures were grown in $10 \mathrm{~mL}$ of YPD medium in a $100 \mathrm{~mL}$ flask. Subsequently the strains were spun down for $1 \mathrm{~min}$ at $14,000 \mathrm{rpm}$. The total RNA was isolated using a Total RNA Mini Plus kit (A\&A Biotechnology, Poland) according to the manufacturer's protocol. Each sample was treated with DNase I (Thermo Scientific) as described in the protocol provided by the company. The RNA quantities were checked by a Biochrom WPA Biowave II spectrophotometer (Biochrom Ltd., UK) equipped with a TrayCell (Hellma Analytics, Germany), then the samples were stored at $-80{ }^{\circ} \mathrm{C}$. The cDNA synthesis was conducted using Maxima First Strand cDNA. The qRT-PCR was analyzed using a DyNAmo Flash SYBR Green qPCR Kit (Thermo Scientific) and the Eco Real-Time PCR System (Illumina, USA). Primers qRPE1F (5'-TCGGCGCACAATCGCGAATG-3) and qRPE1R (5'-GGGCC AAACGAAATGTTGGG-3) resulted in a 97 bp qRT-PCR product. Other primers for RT-PCR were designed as described before [16]. The results were normalized to the actin gene ACT-F/ACT-R and analyzed using the ddCT method. Samples were analyzed in triplicate.

\section{Results and discussion \\ Overexpression of endogenous DGA1 and PPP genes in $Y$. lipolytica during lipogenesis}

In an effort to obtain a high titer of fatty acid we chose a Y. lipolytica wild-type strain named A-101 known for high production of biomass [17]. Next, the strain was modified as described before [18]. Because the DGA1 gene encoding diacylglycerol acyltransferase was found as a key factor of triacylglyceride (TAG) production $[19,20]$, the vector overexpressing the $D G A 1$ gene under the $\mathrm{UAS}_{16} \mathrm{~B}_{16}$-TEF promoter was transformed into it, resulting in strain AJD pAD-DGA1 [13]. Biosynthesis of fatty acid and in consequence synthesis of TAG requires huge quantities of NADPH, since fatty acid are a very reduced form. Previously it was shown that during lipogenesis increased demand for NADPH is fulfilled by the pentose phosphate pathway [21]. For this reason, the vectors harboring overexpression cassettes containing genes encoding ribulose-phosphate 3-epimerase (RPE1, YALI0C11880g), transaldolase (TAL1, YALIOF15587g), transketolase (TKL1, YALIOE06479g), NADP ${ }^{+}$-dependent glucose-6-phosphate dehydrogenase (ZWF1, YALIOE22649g) and 
$\mathrm{NADP}^{+}$-dependent 6-phosphogluconate dehydrogenase (GND1, YALIOB15598g) were introduced to strain AJD pAD-DGA1, resulting in the strains listed in Table 1. The PPP contains two phases, an oxidative and nonoxidative phase (Fig. 1); thus, at the beginning, glucose 6-phosphate is dehydrogenated, by 6-phosphogluconate dehydrogenase, resulting in $\mathrm{NADPH}$ and 6-phosphoglucono- $\delta$-lactone. Consequently, 6-phosphoglucono- $\delta$-lactone is hydrolyzed by a lactonase resulting in 6-phosphogluconate. Next, this compound is oxidatively decarboxylated by 6 -phosphogluconate dehydrogenase, giving ribulose-5-phosphate with cogenerating of NADPH. In the second, nonoxidative phase of PPP, transketolase changes xylose-5-P and ribose-P into glyceraldehyde-3-P (GAP) and sedoheptulose-7-P. Subsequently, these compounds are catalyzed by transaldolase, yielding fructose-6-P and erythrose-4-P. Transketolase converts GAP with fructose-6-P into xylulose-5-P and erythrose-4-P. In parallel, GAP is generated in the second stage of glycolysis and subsequently converted into 1,3-bisphosphoglycerate. Consequently, the glycolysis is finished with pyruvate that links with the TCA cycle in the mitochondria. Produced citrate is transported out to the cytoplasm and is converted into oxaloacetate and a molecule of acetyl-CoA [21], which is a substrate for lipid synthesis. This process occurs in endoplasmic reticulum and the produced lipids are stored in the lipid bodies [2].

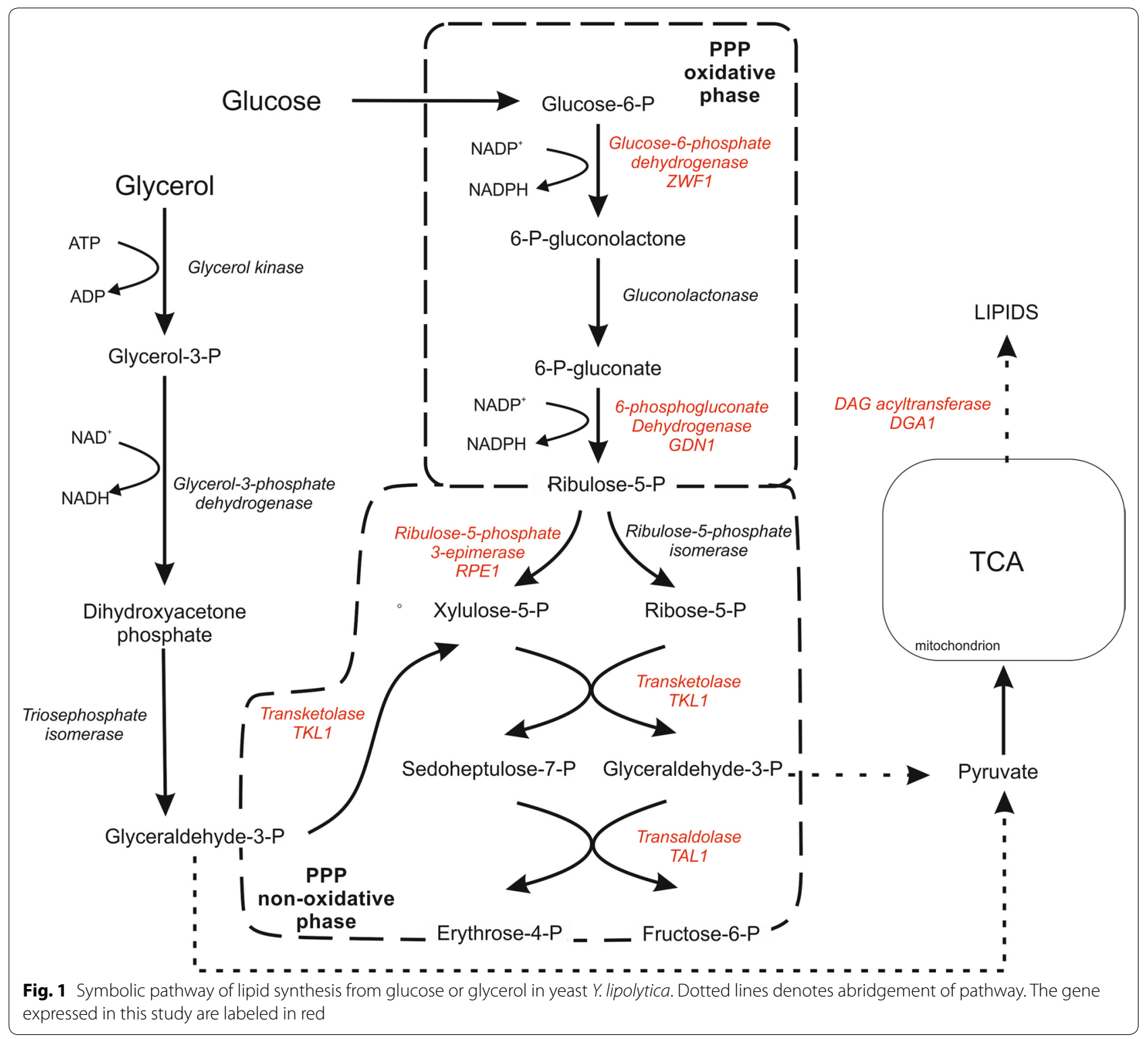


The most common method in metabolic engineering to increase the level of the desired product is functional overexpression of the genes involved in the relevant metabolic pathways. For this reason, the first aim of our study was to evaluate the expression level of the overexpressed genes. For this, we isolated the total RNA from the strains growing for $24 \mathrm{~h}$ on YPD medium. As a control the wild-type strain was used. As seen in Fig. 2, all modified strains showed an elevated gene expression level in comparison to the wild type. Surprisingly, despite the fact that that all overexpression cassettes possess the same hybrid $\mathrm{UAS}_{1 \mathrm{~B}_{16}}$-TEF promoter, the expression level significantly varies among the strains, but the same effect was observed for this promoter before [15, 20, 22]. The highest activity of this promoter was observed after 24 h of growth [23]. Probably, the strains were not synchronized, and thus the promotor activity varied among them. Despite this fact, here we proved that all genes were overexpressed in the modified strains.

\section{Growth of the engineered Y. lipolytica strains}

The metabolic engineering improves the ability of the modified strain to produce the desired metabolites, but sometimes it might impair the functionality of the cell [24]. Therefore, to evaluate whether double overexpression has a negative influence on $Y$. lipolytica growth we performed a growth experiment in a microplate reader. As a carbon source glycerol was used. In this study we employed glycerol as a carbon since it was reported that on this substrate $Y$. lipolytica shows a higher growth rate than on glucose [25] and glycerol is a precursor of triacylglycerols (TAG), the most common form of lipids stored in lipid bodies. As seen in Fig. 3, all the modified strains

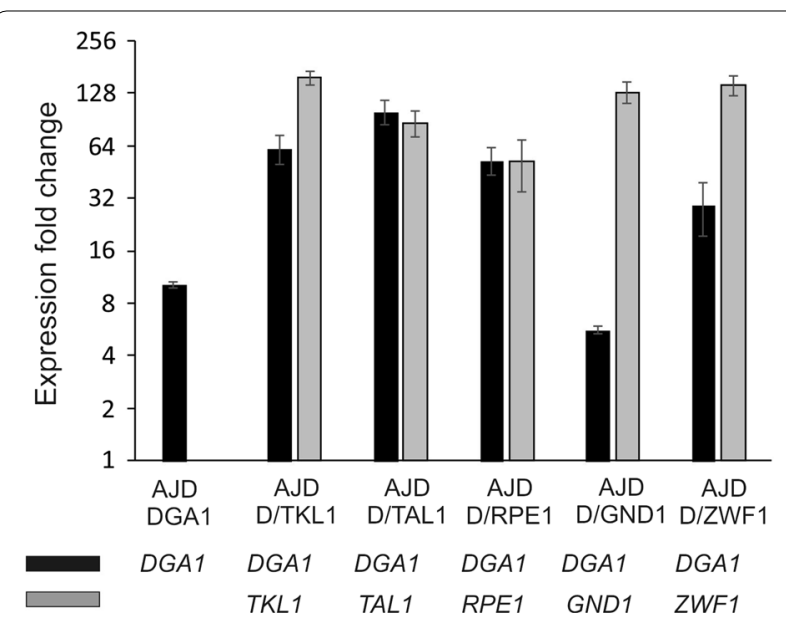

Fig. 2 Expression level of genes overexpressed in this study. The reference gene was actin. The samples were prepared in three repetitions. Error bars represent standard deviation

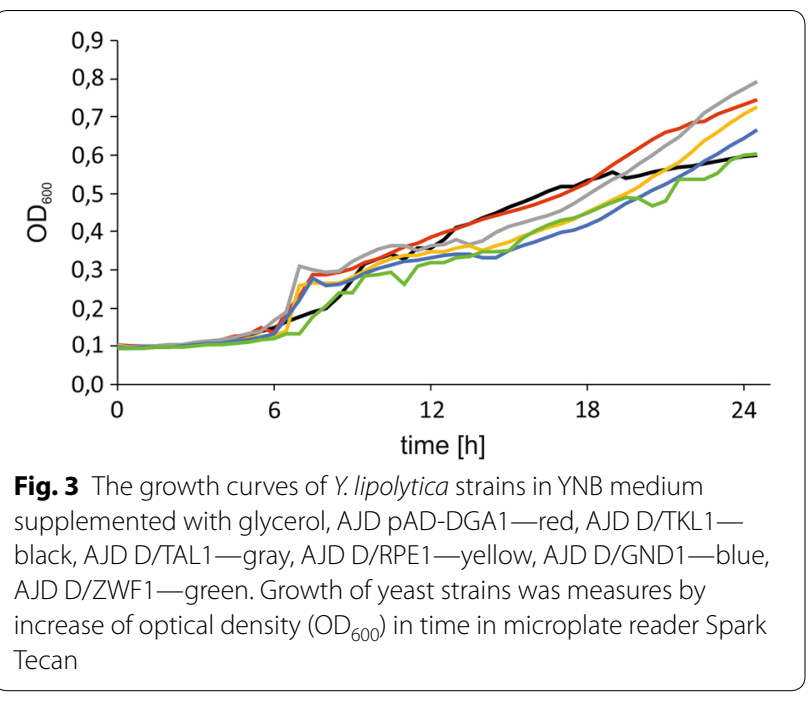

were able to grow in medium supplemented with glycerol, and no significant delay in growth was observed.

Since the genes overexpression was confirmed, we performed three sets of fermentations for all strains: the first on YNB supplemented with glycerol, pH 6.0 (Medium A), the second on YNB supplemented with glycerol, $0.5 \mathrm{~g} / \mathrm{L}$ YE, pH 3.0 (Medium B), and the third on YNB supplemented with glucose, $0.5 \mathrm{~g} / \mathrm{L} \mathrm{YE,} \mathrm{pH} 3.0$ (Medium C). In all cases the ratio $\mathrm{C} / \mathrm{N}$ was 60 .

The results of the fermentations are shown in Fig. 4. The biomass production and fatty acid synthesis of each strain were assessed in terms of lipid titer and cellular lipid content. In Medium A (Fig. 4a), the biomass titer was around $5 \mathrm{~g} / \mathrm{L}$ and the highest biomass was observed for strain AJD D/TKL1 (5.9 g/L). The lowest titer was observed for AJD D/GND1 (2.6 g/L); for the same reason, the lowest lipid titer and content were observed for this strain. The highest lipid synthesis was shown by the strain overexpressing DGA1 and TKL1; the titer was $1.42 \mathrm{~g} / \mathrm{L}$ and content was $23.94 \%$. This is an improvement by $40 \%$ in comparison to the control strain AJD pAD-DGA1 (0.84 g/L, 16.89\%, respectively). Overexpression of other genes did not result in a higher biomass titer or in significantly improved lipid synthesis. Co-expression of DGA1 and ZWF1 or GND1 did not result in a higher lipid titer. This result confirms other research where it was shown that simple overexpression of these genes involved in NADPH synthesis did not result in higher lipid production [15]. Surprisingly, we observed lower biomass production and consequently a lower lipid titer in strain AJD D/GND1; however, this strain showed lower DGA1 expression (Fig. 1), and this might explain this result.

Next, we changed the fermentation conditions and the $\mathrm{pH}$ was decreased to 3.0 and medium was supplemented 
(See figure on next page.)

Fig. 4 Results of fermentations of the engineered strains in three different media after $120 \mathrm{~h}$. a Medium A, YNB supplemented with $50 \mathrm{~g} / \mathrm{L}$ glycerol, pH 6.0. b Medium B, YNB supplemented with $50 \mathrm{~g} / \mathrm{L}$ glycerol, $0.5 \mathrm{~g} / \mathrm{L} \mathrm{YE,} \mathrm{pH} \mathrm{3.0.} \mathrm{c} \mathrm{Medium} \mathrm{C,} \mathrm{YNB} \mathrm{supplemented} \mathrm{with} 50 \mathrm{~g} / \mathrm{L} \mathrm{glucose,} 0.5 \mathrm{~g} / \mathrm{L}$ YE, $\mathrm{pH}$ 3.0. For all media $\mathrm{C} / \mathrm{N}$ ratio was 60 . The obtained values of yeast biomass $(\mathrm{g} / \mathrm{L})$ - green bars, lipid titer $(\mathrm{g} / \mathrm{L})$-red bars and lipid content in DCW (\%) - black bars are presented. The experiment was prepared in triplicate for each strain. Error bars represent standard deviation

with $0.5 \mathrm{~g} / \mathrm{L}$ YE (Medium B). Under these conditions production of citric acid by $Y$. lipolytica is almost completely inhibited [26, 27], and thus the carbon flux is redirected to biomass synthesis. The obtained results confirm this phenomenon. Under these conditions all tested strains showed significantly higher biomass production and it ranged from 7.5 to $9.93 \mathrm{~g} / \mathrm{L}$ (Fig. 4b). Therefore, the fatty acid titer was also higher and the content was improved in comparison to Medium A. Again co-expression of $D G A 1$ and TKL1 resulted in the highest lipid content, reaching $26.16 \%$. Improvement in fatty acid synthesis was shown by strain AJD D/ZWF1 and the content reached 25.84\%. The lowest biomass titer and lipid content were obtained by the strain overexpressing DGA1 and GND1, $7.5 \mathrm{~g} / \mathrm{L}$, and $7.09 \%$, respectively. It is worth noting that for the two first strains DGA1 overexpression was maintained at a high level (63- and 30-fold respectively), whereas for the latter it was significantly lower (4.5-fold). This might explain the ineffective lipid accumulation.

Next, to verify whether addition of yeast extract had a significant influence on biomass and lipid production, we performed the shake flask experiment with the engineered strains in Medium C (glucose was used as a main carbon source). Supplementation with YE was maintained at the same level as in Medium B. The results are shown in Fig. 4c. Surprisingly, production of biomass for all strains was lower than in Medium B; thus addition of YE did not have a strong impact on biomass synthesis. The level of biomass titer ranged from 3.60 to $7.87 \mathrm{~g} / \mathrm{L}$, for AJD D/GND1 and AJD D/TKL1, respectively. Another strain overexpressing DGA1 and the NADPH generation gene, ZWF1, achieved $4.93 \mathrm{~g} / \mathrm{L}$ of biomass and $14.51 \%$ lipid content. Previously it was shown that overexpression of ZWF1 under the same conditions resulted in similar lipid content, but slightly lower biomass production [15]. Most likely, in our study, $D G A 1$ expression improved biomass synthesis, but it did not elevate fatty acid content. The strain overexpressing ribulose-phosphate 3-epimerase (RPE1) showed lower titers for both biomass and fatty acid, as under previous conditions. In Medium C the strain overexpressing TKL1 showed the highest titers for both biomass and lipids, $7.87 \mathrm{~g} / \mathrm{L}$ and $1.50 \mathrm{~g} / \mathrm{L}$ respectively. The lipid content in this strain was $19.17 \%$. As seen in Fig. 4, supplementation with YE did not have a large impact on biomass synthesis or on lipid titer. The most suitable carbon source for lipid production by $Y$. lipolytica is glycerol. Probably the presence of glycerol in medium simplifies the synthesis of TAG since it is a component of triglyceride. Co-expression of DGA1 and TKL1 genes results in the improvement in fatty acid synthesis independently from fermentation conditions.

\section{Fatty acid profile in the engineered strain}

Overexpression of gene influences on the total yield and titer of lipid in cells $[15,28]$ but also fermentation conditions might have an impact on fatty acid composition [5, $29,30]$. In this study in addition to observing the influence on biomass and fatty acid titer, we assessed the influence of PPP genes' overexpression and conditions on fatty acid profile. The results of that analysis carried out on data from fermentation on three media are shown in Fig. 5.

The obtained data showed that for all media the most abundant fatty acid is oleate (C18:1). In Medium A, its content was around $50 \%$ by weight of the total fatty acid level, for all of the strains. The highest concentration was observed for ADJ D/TKL1 (51.5\%), the lowest (40.6\%) in strain AJD D/GDN1 (Fig. 5a). Overexpression of DGA1 in all tested strains resulted in elevated stearate (C18:0) synthesis; it ranged from 9.7 to $16.5 \%$. This phenomenon has been described before [20], since content of stearate in the fatty acid profile in the wild-type strain grown on glycerol is below 5\% [31]. Palmitate was produced at a similar level as stearate and its content was about $12.5 \%$ for all strains. Linoleate (C18:2), which belongs to polyunsaturated fatty acids (PUFAs), was produced at a lower level, and its content was below $10 \%$ with the exception of AJD D/GDN1 (11.9\%) and AJD D/TAL1 (10.8\%).

Interestingly, the change of medium $\mathrm{pH}$ to 3.0 and addition of YE have an influence on fatty acid profile (Fig. 5b). In Medium B, all strains, except AJD D/GND1, showed significantly higher content of stearate; its pool reached $37-40 \%$ by weight of total fatty acid concentration in the engineered strains. Strain AJD D/GND1 accumulated the highest amount of oleate (45.1\%), whereas the other strains accumulated below 34\%. Overexpression of TKL1 did not have an influence on fatty acid profile; it was almost unchanged in comparison to the control strain (AJD pAD-DGA1). A similar effect was observed in Medium C. Under these conditions, all strains accumulated a higher amount of stearate (Fig. 5c). However, 
a 10

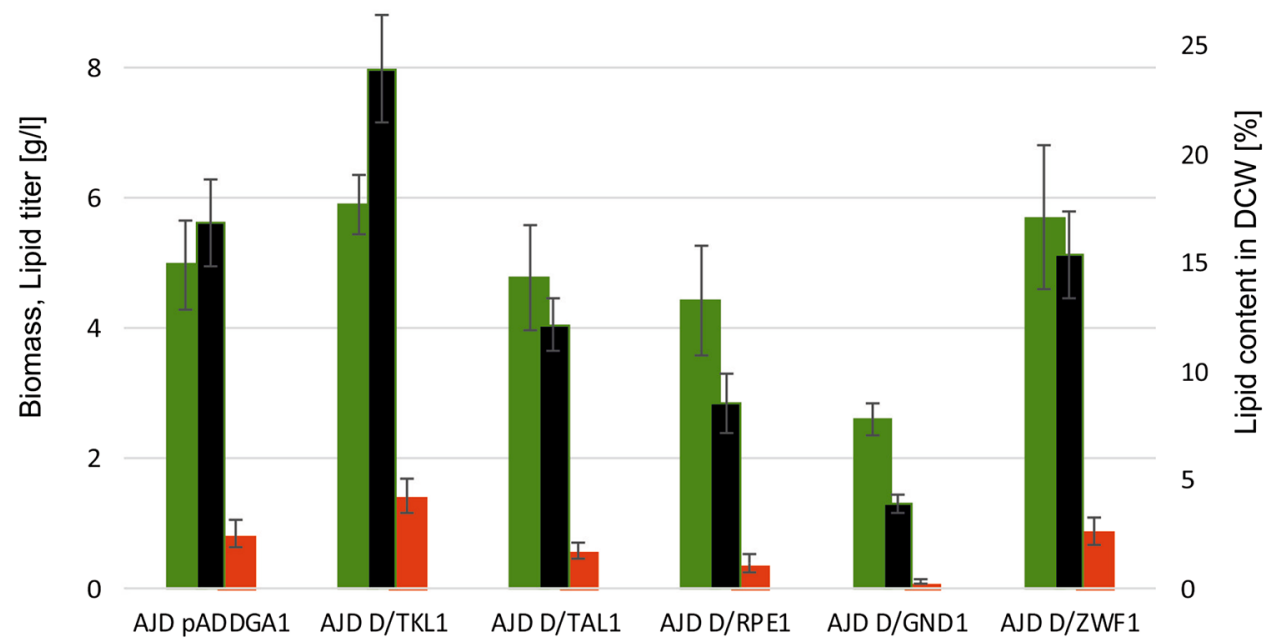

b 12

\section{b 12}

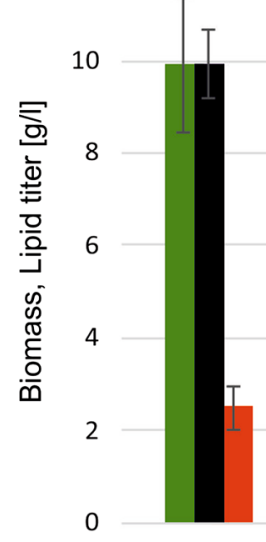

AJD pADDGA1 AJDD/TKL1

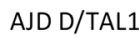

30

25

20

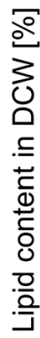

C 10

25

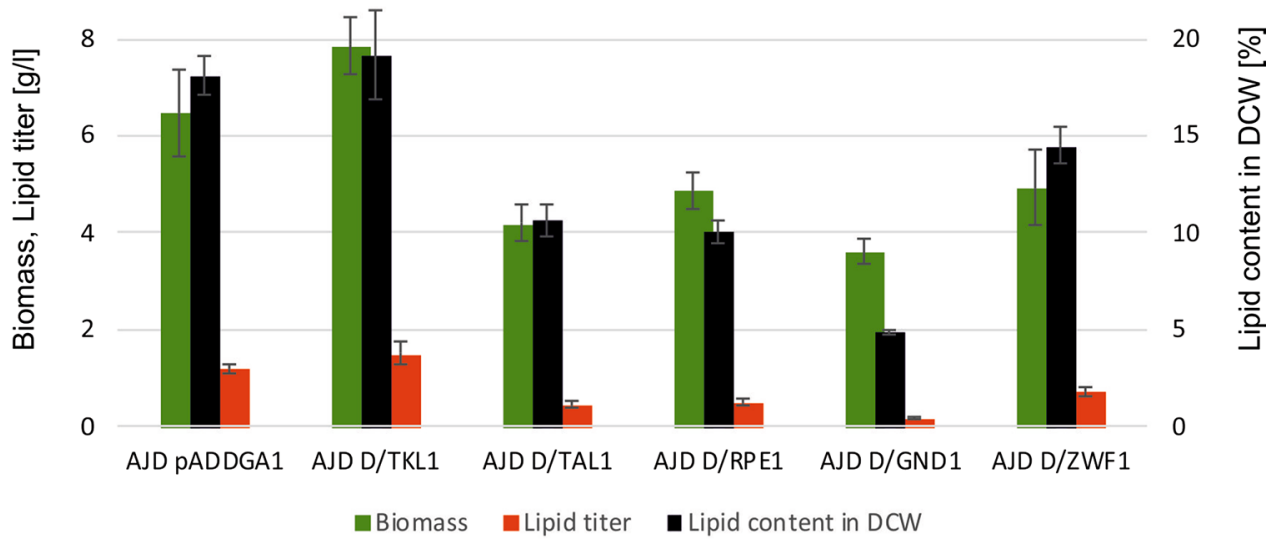




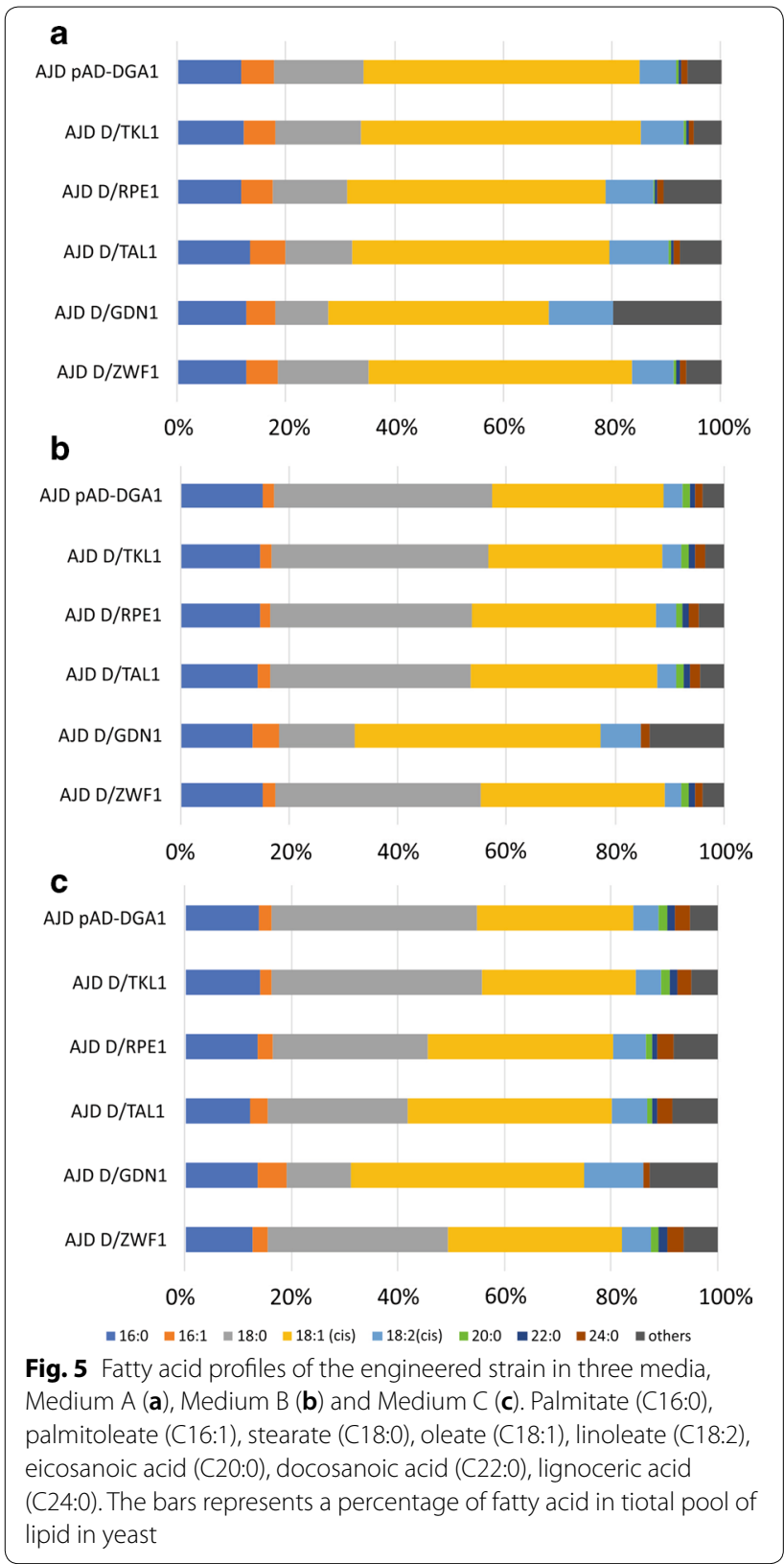

in medium where glucose was the main carbon source, strains AJD D/ZWF1, AJD D/GND1, AJD D/RPE1 and AJD D/TAL produced more oleate $(32.5 \%, 43.5 \%, 38.3 \%$, $34.9 \%$, respectively) than the control strain (29.3\%). Strain AJD D/TKL1 accumulated the highest content of stearate (39.6\%), but its profile was the most similar to the control strain. Moreover, apart from the fermentation conditions, co-overexpression of DGA1 and TKL1 showed the highest content of fatty acid in the biomass. Strain AJD D/TKL1 demonstrated the most similar fatty acid profile to the control strain (AJD pAD-DGA1). Although the overexpression of the other genes investigated here showed a rather modest effect on lipid production, their potential role in this process might be still undiscovered. Since lipid synthesis is a complex process, their function might require other factors, and that might be done by metabolic engineering or fermentation optimization.

\section{Conclusions}

The obtained data showed that co-expression of DGA1 and TKL1 in the yeast Y. lipolytica allows for robust growth of yeast and improvement in fatty acid synthesis. The strain produced $40 \%$ more TAG over the control strain in medium with glycerol as a sole carbon source. Moreover, its fatty acids profile was almost unchanged in comparison to the control strain (DGA1). In addition, these results demonstrate that carbon source and medium supplementation have an influence on fatty acid profile in Y. lipolytica.

\section{Acknowledgements}

The authors thanks Magdalena Marszolik for technical support.

\section{Authors' contributions}

$A D$ designed the study, constructed the strains, participated in fermentation experiments, analyzed the data and wrote the manuscript. AMM performed qRT-PCR, participated in the TECAN experiments, and wrote and revised the manuscript. Both authors read and approved the final manuscript.

\section{Funding}

This work was financially supported by the National Science Centre, Poland, Project 2017/26/E/NZ9/00975. The publication fee is financed under the Leading Reasarch Groups support project from the subsidy increased for the period 2020-2025 in the amount of 2\% of the subsidy referred to Art. 387 (3) of the Law of 20 July 2018 on Higher Education and Science, obtained in 2019.

\section{Availability of supporting data}

The authors promise the availability of supporting data.

Ethical approval and consent to participate Not applicable.

\section{Consent for publication}

The authors have consented for publication.

\section{Competing interests}

The authors declare that they have no competing interests.

Received: 18 April 2020 Accepted: 7 July 2020

Published online: 11 July 2020

\section{References}

1. Dourou M, et al. Critical steps in carbon metabolism affecting lipid accumulation and their regulation in oleaginous microorganisms. Appl Microbiol Biotechnol. 2018;102(6):2509-23.

2. Lazar Z, Liu N, Stephanopoulos G. Holistic approaches in lipid production by Yarrowia lipolytica. Trends Biotechnol. 2018;36(11):1157-70.

3. Kamzolova SV, Morgunov IG. Biosynthesis of pyruvic acid from glycerolcontaining substrates and its regulation in the yeast Yarrowia lipolytica. Bioresour Technol. 2018;266:125-33.

4. Papanikolaou S, et al. Single cell oil production by Yarrowia lipolytica growing on an industrial derivative of animal fat in batch cultures. Appl Microbiol Biotechnol. 2002;58(3):308-12. 
5. Papanikolaou S, et al. Accumulation of a cocoa-butter-like lipid by Yarrowia lipolytica cultivated on agro-industrial residues. Curr Microbiol. 2003:46(2):124-30.

6. Darvishi F, et al. Advances in synthetic biology of oleaginous yeast Yarrowia lipolytica for producing non-native chemicals. Appl Microbiol Biotechnol. 2018;102(14):5925-38.

7. Groenewald M, et al. Yarrowia lipolytica: safety assessment of an oleaginous yeast with a great industrial potential. Crit Rev Microbiol. 2014:40(3):187-206.

8. Stovicek V, Holkenbrink C, Borodina I. CRISPR/Cas system for yeast genome engineering: advances and applications. FEMS Yeast Res. 2017;17(5):fox030

9. Daskalaki A, et al. Laboratory evolution strategies for improving lipid accumulation in Yarrowia lipolytica. Appl Microbiol Biotechnol. 2019;103(20):8585-96.

10. Lazar Z, et al. Hexokinase - a limiting factor in lipid production from fructose in Yarrowia lipolytica. Metab Eng. 2014;26:89-99.

11. Ledesma-Amaro R, Dulermo T, Nicaud JM. Engineering Yarrowia lipolytica to produce biodiesel from raw starch. Biotechnol Biofuels. 2015;8:148.

12. Li H, Alper HS. Enabling xylose utilization in Yarrowia lipolytica for lipid production. Biotechnol J. 2016;11(9):1230-40.

13. Dobrowolski $A$, et al. Lipid production from waste materials in seawaterbased medium by the yeast Yarrowia lipolytica. Front Microbiol. 2019;10:547.

14. Qiao K, et al. Engineering lipid overproduction in the oleaginous yeast Yarrowia lipolytica. Metab Eng. 2015;29:56-65.

15. Silverman AM, et al. Functional overexpression and characterization of lipogenesis-related genes in the oleaginous yeast Yarrowia lipolytica. Appl Microbiol Biotechnol. 2016;100(8):3781-98.

16. Mirończuk AM, Biegalska A, Dobrowolski A. Functional overexpression of genes involved in erythritol synthesis in the yeast Yarrowia lipolytica. Biotechnol Biofuels. 2017;10:77.

17. Wojtatowicz M, Rymowicz W. Effect of inoculum on kinetics and yield of citric acids production on glucose by Yarrowia lipolytica A-101. Acta Aliment Pol. 1991:41(2):137-43.

18. Mirończuk AM, et al. A two-stage fermentation process of erythritol production by yeast $Y$. lipolytica from molasses and glycerol. Bioresour Technol. 2015;198:445-55.

19. Blazeck J, et al. Harnessing Yarrowia lipolytica lipogenesis to create a platform for lipid and biofuel production. Nat Commun. 2014;5:3131.
20. Tai M, Stephanopoulos G. Engineering the push and pull of lipid biosynthesis in oleaginous yeast Yarrowia lipolytica for biofuel production. Metab Eng. 2013;15:1-9.

21. Wasylenko TM, Ahn WS, Stephanopoulos G. The oxidative pentose phosphate pathway is the primary source of NADPH for lipid overproduction from glucose in Yarrowia lipolytica. Metab Eng. 2015;30:27-39.

22. Mirończuk AM, et al. A novel strain of Yarrowia lipolytica as a platform for value-added product synthesis from glycerol. Biotechnol Biofuels. 2016;9(1):1-12.

23. Blazeck J, et al. Generalizing a hybrid synthetic promoter approach in Yarrowia lipolytica. Appl Microbiol Biotechnol. 2013;97(7):3037-52.

24. Kerkhoven EJ, et al. Leucine biosynthesis is involved in regulating high lipid accumulation in Yarrowia lipolytica. MBio. 2017. https://doi. org/10.1128/mBio.00857-17.

25. Workman M, Holt P, Thykaer J. Comparing cellular performance of Yarrowia lipolytica during growth on glucose and glycerol in submerged cultivations. AMB Express. 2013;3(1):58.

26. Mironczuk AM, et al. A novel strain of Yarrowia lipolytica as a platform for value-added product synthesis from glycerol. Biotechnol Biofuels. 2016;9(1):180.

27. Tomaszewska $L$, et al. A comparative study on glycerol metabolism to erythritol and citric acid in Yarrowia lipolytica yeast cells. FEMS Yeast Res. 2014;14(6):966-76.

28. Ledesma-Amaro R, et al. Metabolic engineering of Yarrowia lipolytica to produce chemicals and fuels from xylose. Metab Eng. 2016;38:115-24.

29. Dobrowolski A, et al. Efficient conversion of crude glycerol from various industrial wastes into single cell oil by yeast Yarrowia lipolytica. Bioresour Technol. 2016;207:237-43.

30. Kothri M, et al. Microbial sources of polyunsaturated fatty acids (PUFAs) and the prospect of organic residues and wastes as growth media for PUFA-producing microorganisms. FEMS Microbiol Lett. 2020. https://doi. org/10.1093/femsle/fnaa028.

31. Juszczyk P, et al. Biomass production by novel strains of Yarrowia lipolytica using raw glycerol, derived from biodiesel production. Bioresour Technol. 2013;137:124-31.

\section{Publisher's Note}

Springer Nature remains neutral with regard to jurisdictional claims in published maps and institutional affiliations.
Ready to submit your research? Choose BMC and benefit from:

- fast, convenient online submission

- thorough peer review by experienced researchers in your field

- rapid publication on acceptance

- support for research data, including large and complex data types

- gold Open Access which fosters wider collaboration and increased citations

- maximum visibility for your research: over $100 \mathrm{M}$ website views per year

At BMC, research is always in progress.

Learn more biomedcentral.com/submissions 